\title{
Transmission congestion management considering multiple and optimal capacity DGs
}

\author{
Rajagopal PEESAPATI ${ }^{1}$, Vinod Kumar YADAV ${ }^{2}$, Niranjan KUMAR ${ }^{1}$
}

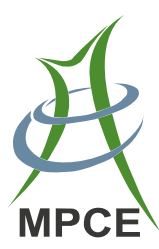

\begin{abstract}
Transmission congestion management became a grievous issue with the increase of competitiveness in the power systems. Competitiveness arises due to restructuring of the utilities along with the penetration of auxiliary services. The present study depicts a multi objective technique for achieving the optimal capacities of distributed generators (DG) such as solar, wind and biomass in order to relieve congestion in the transmission lines. Objectives like transmission congestion, real power loss, voltages and investment costs are considered to improve the technical and economical performances of the network. Multi objective particle swarm optimization algorithm is utilized to achieve the optimal sizes of unity power factor DG units. The insisted methodology is practiced on IEEE-30 and IEEE-118 bus systems to check the practical feasibility. The results of the proposed approach are compared with the genetic algorithm for both single and multi-objective cases. Results revealed that the intimated method can aid independent system operator to remove the burden from
\end{abstract}

CrossCheck date: 16 January 2017

Received: 19 April 2016/Accepted: 16 January 2017/Published online: 19 April 2017

(C) The Author(s) 2017. This article is an open access publication

$\triangle$ Rajagopal PEESAPATI

gopal.12rsee009@nitjsr.ac.in

Vinod Kumar YADAV

v3k5y7@gmail.com

Niranjan KUMAR

nkumar.ee@nitjsr.ac.in

1 Department of Electrical Engineering, National Institute of Technology Jamshedpur, Jamshedpur, Jharkhand 831014, India

2 Department of Electrical Engineering, Gautam Buddha University, Noida 201334, India lines in the contingency conditions in an optimal manner along with the improvement in voltages and a reduction in real power losses of the network.

Keywords Transmission congestion, Optimal power flow, Distributed generation, Particle swarm optimization

\section{Introduction}

In the present restructured environment, new technologies are employed on electric utilities to gain maximum profits while supplying reliable power to the consumers. Unbundling of vertically integrated power system into various sectors like generation, transmission and distribution, exposes the market for a variety of services provided by the utilities. This disintegration converts the monopolistic behavior of electricity markets into a competitive one. Uncertainties like imperfect scheduling of generators and transmission line contingencies should be conquered and relieved at the earliest to make optimum utilization of available transmission network in order to achieve maximum profits $[1,2]$.

Generator rescheduling and load shedding are proposed as the control actions for alleviating the congestion in the network lines. In this view, a method based on local optimization is employed to achieve the best solution [3]. In addition, sensitivities of the overload lines with respect to the bus injections are also considered along with the previous mechanisms [4]. Similarly, objectives like over load alleviation and production cost minimization are considered in a multi-objective based TCM study. PSO based solution methodology is utilized in achieving the global optimal values [5]. In [6], clustering algorithm is utilized to identify the most sensitive zones for congestion employing the real and reactive transmission congestion distribution factors. 
Later, the problem of TCM is limited to generator rescheduling only. In this, rescheduling cost is minimized while managing congestion by altering the output levels of the participating generators which are selected based on generator sensitivity factors. Many algorithms have been proposed to alter the preferred active power generations for alleviating congestion in the network lines. Initially, PSO is proposed to reschedule the outputs of participating generators to obtain the minimum deviations [7]. Further, Adaptive bacterial foraging algorithm with Nelder-Mead (ABFNM) is employed to minimize the congestion (rescheduling) cost of standard IEEE-30 bus system [8].

Many procedures reveal the usage of static VAR compensators and flexible ac transmission systems (FACTS) for the congestion management problem. In this, Benders decomposition is utilized as an efficient algorithm to place the static VAR compensators and FACTS devices optimally for relieving congestion in the lines $[9,10]$. In [11], the TCM problem is solved using a qualitative bidding strategy which is achieved through a dynamic game. Rescheduling of generator outputs and transaction curtailments together are employed to manage the congestion that is caused due to bi-lateral and multi-lateral transactions [12]. Similarly, three different frameworks Monte Carlo Simulation (MCS), Lattice Rank-1 MCS and Lattice Rank2 MCS are employed to control the congested line power flows [13].

On the other hand, due to heavy competition in the electricity markets load side governing is more preferred instead of supply side governing in the case of TCM [14]. In this regard, auxiliary services are penetrated in the present competitive electricity markets to improve reliability and security of the power system [15]. Auxiliary services like distributed generators in addition with the conventional generators provide better stand in loss reduction, voltages improvement and congestion relieving. The most proven DGs are diesel, solar thermal systems, biomass and wind.

Lot of research works reveals the application of DGs in distribution systems for voltage improvement and reducing real power losses thereby enhancing the performance of the network. Artificial intelligence based solution methods are applied to obtain the optimal capacities using single and multi-objective functions. Objectives like power flows, loss reduction, voltage improvement and cost factors are considered simultaneously to obtain the optimal capacity DGs [16].

Many researches in the past utilized various artificial intelligence and sensitivity based techniques for obtaining the optimal places and capacities of DGs to be integrated with the distribution network. In view of this, sensitivities based on real and reactive power losses are evaluated to obtain the size of DG in a weakly mesh distribution network [17]. Hybrid PSO (HPSO) is employed to minimize the system loadability for obtaining the optimal place and sizes of DGs for various radial test bus systems [18]. Weighted aggregation PSO (WAPSO) is employed to obtain the size, type and location of DG to be connected with the practical Indian distribution systems [19]. Many algorithms like fireworks optimization algorithm [20] and bacterial foraging algorithm (BFA) [21] are also implemented to obtain DGs placement and their capacities. Some of the researches concentrated on reducing the search space for the above mentioned procedures [22]. A method based on network reconfiguration along with DG insertion is proposed in [23]. Later, the results were further improved with the implementation of improved bat algorithm (BAT) [24].

DGs are also integrated in transmission networks to control the power flows and to increase the performance of the network. $Z_{B U S}$ based contribution factors are determined to optimally insert the DGs for the TCM problem. A $60 \mathrm{MW}$ DG was inserted at the optimal location to alleviate the congestion in the line 1-3 [25] of standard IEEE 30-Bus system. Likewise, genetic algorithms (GA) are applied to determine the optimal capacities for the TCM problem by considering voltage improvement factors and real power loss reduction factors [26, 27]. LMP based DG integration is proposed for TCM problem [28]. Many random search methods, such as genetic algorithms (GA) and simulated annealing (SA) have recently received much interest for obtaining the optimal capacities of DGs in the distribution systems and transmission systems. Although GA has been successfully employed to complex optimization problems, recent researches revealed some deficiencies in the performance and its search capability when it was presented before highly correlated objective function [29].

So far, many of the researches aimed to obtain the optimal capacity DGs comprising of either only technical $[15,26]$ or only economical factors for the TCM problem [28]. Also, the researches in the past did not adopt the weight selection strategies considering multi objectives [26] and aim the optimal capacity DGs for the TCM problem [25]. The optimal sizing of DGs that are obtained by considering technical factors may direct towards the higher investment costs of DG with a little improvement in the technical performances of the system and may become financial burden which leads to economical infeasibility. Similarly, in case of considering only economical factors, the technical performance may be degraded and may not fulfill the present and future transaction curtailments. Hence, to overcome the above research gaps, in this work authors aimed to obtain the optimal capacities of DGs to improve technical performance along with optimal investment on DG units, which is an important and a new contribution to this field. 
In this work, the demand side management based multiobjective technique is proposed for the TCM problem. Objectives like transmission congestion, voltage improvement, real power loss reduction and investment cost are considered simultaneously by including them with normalized weighting factors to achieve the optimal sizes of DGs. The results of proposed multi-objective optimization problem are evaluated by the application of both GA and PSO for various single and multi-objective cases.

The timeline of the paper is as follows: Sect. 2 shows the formulations for optimal power flow, congestion management problem and artificial intelligence methodology. Section 3 describes the step by step procedure for solving the TCM problem. The results obtained are presented in Sect. 4. Finally, conclusions are deducted in Sect. 5.

\section{Problem formulation}

\subsection{Optimal power flow (OPF)}

In a deregulated environment, the OPF problem is structured by minimizing production cost of generators subjected to power balance constraints and line flow constraints [30]. The OPF problem can be mathematically formulated as:

$\min C=\sum_{i=1}^{N_{g}} F_{g i}$

Subjected to:

1) Power balance constraints:

$$
\begin{gathered}
P_{g i}=P_{d i}+\sum_{j=1}^{N}\left|V_{i}\right|\left|V_{j}\right|\left(G_{i j} \cos \delta_{i j}+B_{i j} \sin \delta_{i j}\right) \\
\forall i=1,2, \ldots, N
\end{gathered}
$$

where $P_{d i}, P_{g i}$ are real power demand and generation at $i^{t h}$ bus; $\delta_{i j}$ is $\delta_{j}-\delta_{i} ; N$ is total number of buses.

$$
\begin{gathered}
Q_{g i}=Q_{d i}+\sum_{j=1}^{N}\left|V_{i}\right|\left|V_{j}\right|\left(G_{i j} \sin \delta_{i j}-B_{i j} \cos \delta_{i j}\right) \\
\forall i=1,2, \ldots, N
\end{gathered}
$$

where $G_{i j}$ and $B_{i j}$ are the conductance and susceptances of the line $i-j$ respectively.

2) Power flow constraints:

$\left|P l_{i j}\right| \leq P l_{i j}^{\max } \quad \forall i j \in N_{l}$

where $P l_{i j}, P l_{i j}^{\max }$ are power flow in the line $i-j$ and its maximum limit respectively; $N_{l}$ is total number of lines.

3) Other in-equality constraints:

$$
\begin{aligned}
& P_{g i}^{\min } \leq P_{g i} \leq P_{g i}^{\max } \quad \forall i=1,2, \ldots, N_{g} \\
& \delta_{i}^{\min } \leq \delta_{i} \leq \delta_{i}^{\max } \quad \forall i=1,2, \ldots, N \\
& V_{i}^{\min } \leq V_{i} \leq V_{i}^{\max } \quad \forall i=1,2, \ldots, N
\end{aligned}
$$

where $P_{g i}^{\min }, P_{g i}^{\max }$ are minimum and maximum limit of $i^{\text {th }}$ generator respectively; $\delta_{i}, \delta_{i}^{\min }, \delta_{i}^{\max }$ are voltage angel of $i^{\text {th }}$ bus and its minimum maximum limits respectively; $N_{g}$ is total number of generators. The production function $(\$ / \mathrm{h})$ of $i^{t h}$ generator can be mathematically represented by

$F_{g i}=\frac{1}{2} a_{g i} P_{g i}^{2}+b_{g i} P_{g i}+c_{g i}$

where $a_{g i}, b_{g i}$ and $c_{g i}$ are the fuel cost coefficients of $i^{t h}$ generation company.

In this work all the inequality constraints are transformed to the penalty functions and are added to the main objective function to construct the final fitness function to be minimized.

\subsection{Congestion management problem with optimal capacity DGs considering economical factors}

The main causes of congestion are line outages, sudden increase in load, reduction in thermal limits and due to a combination of bi-lateral and multi-lateral transactions. Once congestion occurs, independent system operator (ISO) follows various procedures to alleviate the extra power flows in the transmission lines. Various procedures like Generators active power rescheduling, load shedding, insertion of FACT devices and insertion of optimal capacity distributed generators are mainly utilized. In this work, the optimal capacity distributed generators are inserted for TCM problem. After deciding the optimal locations for placing the DGs, TCM problem is formulated to incorporate the effect of DGs. To include the effect of DGs by considering the economic factors, the objective function (1) is altered as follow:

$\min C=\sum_{i=1}^{N_{g}} F_{g i}+\sum_{i=1}^{N_{D G}} F_{D G, i}$

In (9), $F_{D G, i}$ in $(\$ / \mathrm{h})$ is the production cost function of $i^{\text {th }} \mathrm{DG}$ obtained from the slope $a_{D G, i}$ and intercept $b_{D G, i}$ of DG offer as shown below:

$F_{D G, i}=\frac{1}{2} a_{D G, i} P_{D G, i}^{2}+b_{D G, i} P_{D G, i}$

Further, the constraint (2) is also altered to involve the effect of DG and represented as follow: 


$$
\begin{gathered}
P_{g i}=P_{d i}+\sum_{j=1}^{N}\left|V_{i}\right|\left|V_{j}\right|\left(G_{i j} \cos \delta_{i j}+B_{i j} \sin \delta_{i j}\right) \\
\forall i=1,2, \ldots, N, i \neq k \\
P_{g i}+P_{D G, k}=P_{d i}+\sum_{j=1}^{N}\left|V_{i}\right|\left|V_{j}\right|\left(G_{i j} \cos \delta_{i j}+B_{i j} \sin \delta_{i j}\right) \\
i=k
\end{gathered}
$$

The limits on DGs active power generation is also included along with conventional generators limits.

$0 \leq P_{D G, k} \leq P_{D G}^{\max }$

where $P_{D G}^{\max }$ is maximum penetration of $k^{\text {th }} \mathrm{DG}$.

The TCM problem with DG consists of objective function (9) subjected to constraints given by (3)-(7) and (11)-(13). The maximum power delivered by DG units is restricted to its installed capacity for operating the power system during the congestion hours. The maximum capacity of DG is taken as $20 \%$ of the systems total demand. The DGs are inserted as negative power injections at the load pockets during the load flow analysis.

\subsection{Congestion management problem with optimal capacity DGs considering technical and economical factors}

In the present work congestion management with voltage and real power losses improvement are considered as technical factors and production costs of both distributed and conventional generators are considered as economical factor for obtaining the optimum capacities of DG units. The following sub-section shows the various factors that are considered in this work.

\subsubsection{Transmission congestion}

Congestion relieving is the main aim of present work. In view of this, a factor resembling the ratio of the real power flowing through the line after the placement of DG(s) to its line limits is computed for all the transmission lines. The highest of all such fractions is considered as transmission congestion factor (TC). It can be mathematically represented as follow:

$T C=200 \cdot \max \left(\frac{P l_{i j}^{D G}}{P l_{i j}^{\max }}\right)$

where $P l_{i j}^{D G}$ and $P l_{i j}^{\max }$ are the real power flows in the line $i-j$ after including DG in network and the line limiting capacity respectively.

\subsubsection{Voltage improvement (VD)}

The ratio between the sum of squares of the deviations of voltage magnitudes from 1 p.u. before and after including DG in the network is considered as voltage improvement factor. This is mathematically defined as follow:

$V D=200 \cdot \frac{\sum_{i=1}^{N}\left(V_{i}^{D G}-1.0\right)^{2}}{\sum_{i=1}^{N}\left(V_{i, 0}-1.0\right)^{2}}$

where $V_{i}^{D G}$ is voltage magnitude of $i^{\text {th }}$ bus after placement of DG; $V_{i, 0}$ is voltage magnitude of $i^{\text {th }}$ bus before the placement of DG.

\subsubsection{Real power loss (RPL)}

The purpose of considering this parameter is to reduce the real power loss in the power system network. This can be mathematically defined as:

$R P L=200 \cdot \frac{P_{\text {Loss }}^{D G}}{P_{\text {Loss }}^{0}}$

where $P_{L o s s}^{D G}$ is real power loss after including the DG; $P_{\text {Loss }}^{0}$ is real power loss before including the DG.

\subsubsection{Cost factor (CF)}

The main aim of the $C F$ is to obtain the optimum investment costs of all the three types of DGs and conventional generators simultaneously. This is mathematically defined as:

$C F=\sum_{i=1}^{N_{g}} F_{g i}+\sum_{i=1}^{N_{D G}} F_{D G, i}$

\subsection{Mathematical modeling for achieving optimum sizing of DGs}

In this work the problem of optimal sizing of DGs is intended to improve the technical performance along with optimal investments on DG units. In the present multi-objective approach, weighted technical parameters and economical parameters are considered simultaneously to achieve the sizes of DGs. Computation of multi-objective solutions without weight selection strategy may lead to inappropriate solution [26].

In recent, the weights for respective objectives were selected based on the "hypothesis of relevance of objectives" which is deducted from planner's experience. However the process is not methodical and may misguide the entire planning process $[18,31]$. Hence in this work all the objectives are considered simultaneously for the optimization by using the weighting factors for the incorporation of 
DGs with the aim of improving technical and economical performances of transmission network [16]. The multiobjective function for obtaining optimum sizing of DGs is formulated by combining the technical and economical parameters with the weighting factors represented as follow:

$J=h_{1} \cdot T C+h_{2} \cdot V D+h_{3} \cdot R P L+h_{4} \cdot C F$

where $h_{1}, h_{2}, h_{3}$ and $h_{4}$ are the weighting factors of $T C, V D$, $R P L$ and $C F$ respectively.

The multi-objective minimization problem is formulated to minimize the function $J$ (18) subject to the constraints mentioned in (3)-(7), (11)-(13) and an extra constraint as follow:

$\sum_{i=1}^{4} h_{i}=1 \quad h_{i} \in\left[\begin{array}{ll}0 & 1\end{array}\right]$

\subsection{Optimum sizing of DGs using particle swarm optimization}

The classical particle swarm optimization (PSO) technique used for optimizing complex objective functions is first introduced by Kennedy and Eberhart [32]. This is kind of evolutionary computation technique has wide applications in solving problems constituting nonlinearity, nondifferentiability, multiple optima and high dimensionality [29]. PSO is based on the parallel exploration of the search space by a swarm-a set of "particles", the solutions or alternatives. Its key concept is that the potential solutions are flown through search space and are accelerated towards better or more optimum solutions. Every particle in PSO is associated with two vectors, position vector and velocity vector. The position vector of $p^{\text {th }}$ particle is represented as $z_{p}=\left(z_{p 1}, z_{p 2}, \ldots, z_{p d}\right)$ and velocity vector of $p^{\text {th }}$ particle is represented as $v_{p}=\left(v_{p 1}, v_{p 2}, \ldots, v_{p d}\right)$. PSO starts with a population of random solution "particles" in a $d$ dimension space. Each particle preserves the best solution and its respective coordinates so far achieved. The best solution is called $F_{\text {best }}$ and the coordinates associated with best solution are called $P_{\text {best }}$. The best previous positions of the particles in every iteration are recorded in $P_{\text {best, } p}=(-$ $\left.P_{\text {best }, p 1}, P_{\text {best }, p 2}, \ldots, P_{\text {best }, p d}\right)$. The PSO algorithm stores the overall best value and its respective coordinates as $G_{\text {best }}$. The optimization technique updates its velocity and position of each particle at every step towards $P_{\text {best }}$ and $G_{\text {best }}$ to obtain the best solution so far. The particles in the swarm are updated iteratively according to the equations as follow:

$$
\begin{aligned}
v_{p d}^{j+1}= & u \cdot v_{p d}^{j}+c_{1} \cdot \operatorname{rand}_{1} \cdot\left(P_{\text {best }, p d}-x_{p d}^{t}\right) \\
& +c_{2} \cdot \operatorname{rand}_{2} \cdot\left(G_{\text {best }, p}-x_{p d}^{t}\right) \\
z_{p d}^{j+1}= & z_{p d}^{j}+v_{p d}^{j+1}
\end{aligned}
$$

where $n$ is number of particles; $m$ is number of members; $j$ is iteration count; $v_{p d}^{j}$ is velocity of $p^{t h}$ particle at $j^{\text {th }}$ iteration; $u$ is inertia weight vector; $c_{1}$ and $c_{2}$ are acceleration constants; rand $_{1}$ and rand $_{2}$ are random numbers between $0 \& 1 ; z_{p d}^{j}$ is position of $p^{\text {th }}$ particle at $j^{\text {th }}$ iteration; $P_{\text {best,pd }}$ is local best of $p^{\text {th }}$ particle; $G_{b e s t, p}$ is global best of $p^{\text {th }}$ particle.

The weighting factor is evaluated based on the equation as follow:

$u=u_{\max }-\frac{u_{\max }-u_{\min }}{N_{\text {iter }, \max }} \cdot N_{\text {iter }}$

where $u_{\max }$ and $u_{\min }$ are maximum and minimum value of inertia weight respectively; $N_{\text {iter }}$ is iteration count; $N_{\text {iter, } \text { max }}$ is maximum number of iterations.

\section{Flow chart for proposed methodology}

The proposed method aims to achieve the optimal capacity DG units in order to relieve congestion in transmission lines along with improvement and both real power losses and voltages of the network. The optimal capacities of the DG units are achieved by implementing MO-PSO approach. The flow chart for proposed MO-PSO approach is shown in Fig. 1.

\section{Result analysis}

In this work, optimal capacities of DGs are obtained based on the multi-objective approach in order to relieve the congestion in transmission lines of the network. The effectiveness of the proposed approach is examined on IEEE-30 and IEEE-118 bus systems. To check the suitability of proposed approach, case studies including single objective and multi-objectives are considered separately. The case studies in terms of single and multi-objective are shown in Table 1.

The locations of DGs are selected based on $Z_{B U S}$ based contribution factors which was proposed in [25]. Three types of DGs like Solar, Wind and Biomass are considered and their production cost functions are modeled as $a_{D G, i}=0$ and $b_{D G, i}=30(\$ / \mathrm{MWh})$ throughout the work. Following assumptions are made in order to continue the work.

1) Only one DG is connected to the same bus at a time [19].

2) Similarly, the system considered for the study is operated in balanced mode [19]. 


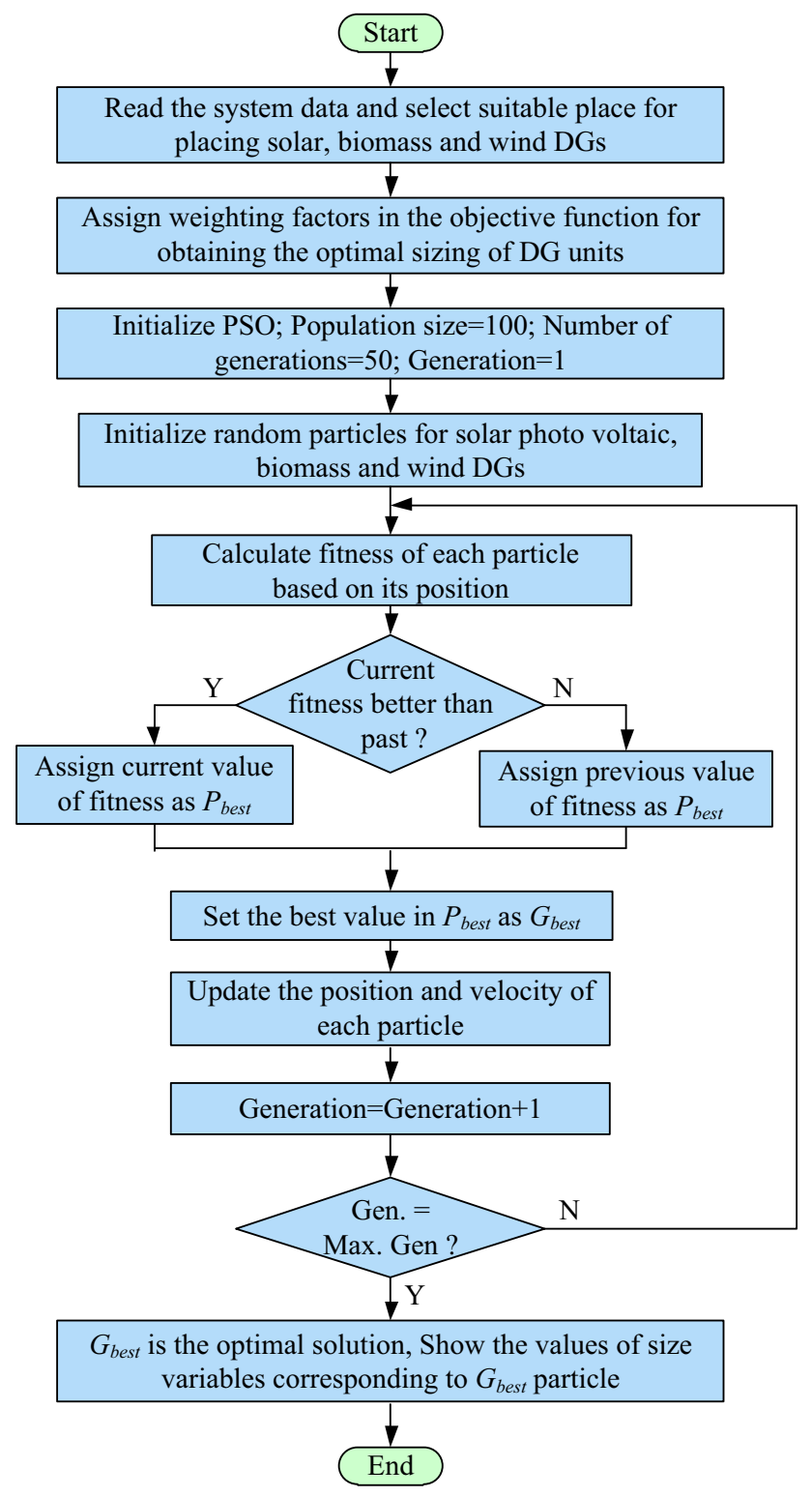

Fig. 1 Flow chart for proposed approach

The optimal capacities of DGs are achieved based on the proposed MO-PSO approach and the results obtained are compared with results of genetic algorithm (GA) and method reported in [25]. The control parameters for both PSO and GA approaches are depicted in Table 2.
Table 2 Control parameters for GA and PSO

\begin{tabular}{ll}
\hline GA & PSO \\
\hline Maximum iteration $=100$ & Maximum iteration $=100$ \\
Population size $=20$ & Population size $=20$ \\
Stall generation limit $=100$ & $c_{1}=c_{2}=2$ \\
& $u_{\max }=0.9, u_{\min }=0.4[29]$ \\
\hline
\end{tabular}

\subsection{Case 1: IEEE-30 bus test system}

The proposed approach is evaluated on standard IEEE30 bus system which has 6 generators, 21 loads and 41 transmission line sections. The generators cost coefficients, their maximum and minimum limits, bus and line data related to the IEEE-30 bus can be obtained from [8]. In Case 1A, the considered network has no DG and the base MVA is considered as $100 \mathrm{MVA}$. OPF is performed to obtain the power flows in the lines, voltages at buses and the real power losses. As bus 1 is reference, the output of all generators except at bus 1 are considered as variables for the OPF run. The results of OPF are shown in Table 3. It is clear that the total fuel cost obtained using proposed approach is $801.8437(\$ / \mathrm{h})$ which is minimum as compared to the GA.

The contingency scenario is created by reducing the line limit of the line $1-3$ from 75 to $60 \mathrm{MW}$. This implies the line $1-3$ is loaded with more $5.65 \%$ of its maximum line loading. Hence in this work, multiple DGs with their optimal capacities are inserted to mitigate the congestion in the line. For the present case study $60 \mathrm{MW}$ is considered as maximum limit of DG $\left(P_{D G}^{\max }\right)$ during the run of reported algorithms. The preferable locations for inserting the optimal capacity DGs with respect to the congested line 1-3 are shown in Table 4. The optimal capacities of DGs at the top three locations are obtained using PSO and GA with the proposed multi-objective function described in (18).

During Case 1C, the objective function is formed with the cost factors of the both conventional and distributed generators. Hence in this case, the weighting factor is set to 1 for both the cost factors of DGs and the conventional generators. The results obtained through reported

Table 1 Case studies considered for present study

\begin{tabular}{llll}
\hline Test case & Test system & Description & Form of objective \\
\hline Case $1 \mathrm{~A} / 2 \mathrm{~A}$ & IEEE-30/118 bus & Without DG & Single \\
Case 1B/2B & IEEE-30/118 bus & With 60 MW DG [25] & Single \\
Case 1C/2C & IEEE-30/118 bus & With optimal capacity DGs with only CF & Single \\
Case 1D/2D & IEEE-30/118 bus & With optimal capacity DGs with all factors & Multi \\
\hline
\end{tabular}


Table 3 Results of OPF according to Case 1A for IEEE-30 bus system

\begin{tabular}{lll}
\hline Generator number & PSO (MW) & GA (MW) \\
\hline 1 & 176.6624 & 177.217 \\
2 & 48.8103 & 48.471 \\
3 & 21.4607 & 21.588 \\
4 & 21.7339 & 21.9621 \\
5 & 12.1028 & 11.882 \\
6 & 12.0 & 12.0 \\
Generation cost $(\$ / h)$ & 801.8437 & 803.032 \\
Losses (MW) & 8.89 & 9.3507 \\
Power flow in line 1-3 (MW) & 63.36 & 62.21 \\
\hline
\end{tabular}

Table 4 Optimal locations for inserting DGs in IEEE-30 bus system

\begin{tabular}{lll}
\hline Bus number & Description & Type of DG \\
\hline 3 & Optimal & Solar \\
4 & Sub-optimal & Biomass \\
13 & Sub-optimal & Wind \\
12 & Sub-optimal & - \\
14 & Sub-optimal & - \\
\hline
\end{tabular}

Table 5 Sizing of DGs in various case studies for IEEE-30 bus system

\begin{tabular}{lllllll}
\hline Bus number & $\begin{array}{l}\text { Case 1B } \\
(\mathrm{MW})\end{array}$ & \multicolumn{2}{l}{ Case 1C } & & \multicolumn{2}{l}{ Case 1D } \\
\cline { 3 - 4 } \cline { 6 - 7 } & & $\begin{array}{l}\text { GA } \\
(\mathrm{MW})\end{array}$ & $\begin{array}{l}\text { PSO } \\
(\mathrm{MW})\end{array}$ & & $\begin{array}{l}\text { GA } \\
(\mathrm{MW})\end{array}$ & $\begin{array}{l}\text { PSO } \\
(\mathrm{MW})\end{array}$ \\
\hline 3 (Solar) & 60 & 19.952 & 8.1647 & & 10.098 & 2.073 \\
4 (Wind) & - & 39.868 & 0 & & 8.728 & 3.374 \\
13 (Biomass) & - & 0.099 & 0 & & 1.044 & 4.517 \\
$\begin{array}{l}\text { Total DG } \\
\text { capacity }\end{array}$ & 60 & 59.199 & 8.1647 & 19.87 & 9.964 \\
\hline
\end{tabular}

approaches during this case study are shown in Table 5 . The total DG power injection using PSO is $8.1647 \mathrm{MW}$, whereas it is $59.91 \mathrm{MW}$ by GA. After the insertion of optimal capacity DGs at suitable sites it has been observed that the power flow in the congested line 1-3 is reduced by 8.3, 11.1 and $5.3 \%$ of its maximum line limit by PSO, GA and according to Case $1 \mathrm{~B}$ respectively. The convergence curves of PSO and GA approaches during Case 1C run are shown in Fig. 2. Form the figure it is clear that the PSO approach converges in less number of iterations and gives better solution as compared to GA.

Similarly, in Case 1D, the weighting factors are kept at 0.25 for each of the objectives in order to have equal weightage during the execution. The optimal capacities

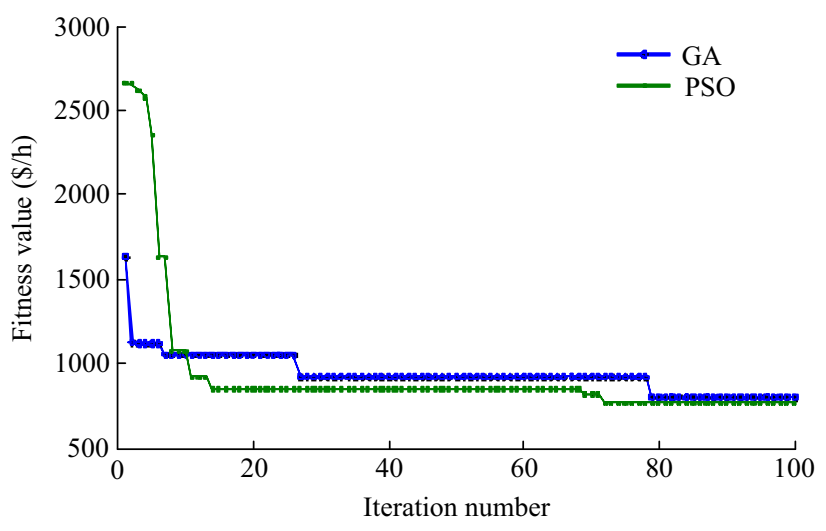

Fig. 2 Convergence curve in Case 1C for IEEE-30 bus system

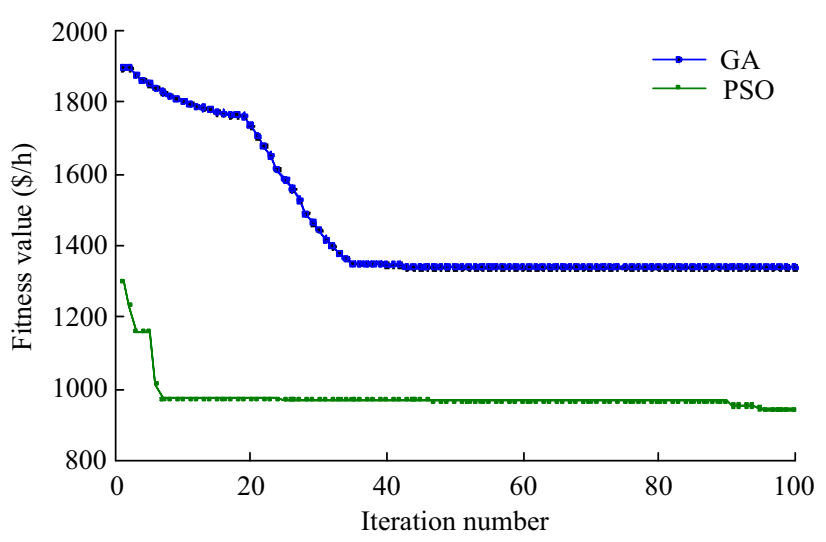

Fig. 3 Convergence curve in Case 1D for IEEE-30 bus system

that are obtained during this case are presented in the Table 5. The convergence curves of both PSO and GA approaches during Case 1D run are shown in Fig. 3. In this case, the total DG power penetration is increased to 9.964 MW by the proposed PSO approach whereas; it is reduced to $19.87 \mathrm{MW}$ by GA. The result obtained after inserting the optimal capacity DGs to the network are presented in Table 6. From the table it is noticed that, the power flows and real power losses in Case 1D have been further decreased by the proposed multi-objective approach as compared to the other single objective approaches.

A comparison with the similar researches in terms of real power flow, real power losses, maximum and minimum values of voltages have been shown in Table 6. Large decrement is observed in the real power losses before and after the placement of the optimum size of DGs. It is noticed that, the power flow in the congested line has been reduced but the real power losses have been increased in Case 1B. Figure 4 shows the voltage magnitudes of each bus in p.u. for different case studies. It discloses that the voltage magnitudes have been improved greatly after 
Table 6 Comparative results of various approaches in different case studies for IEEE-30 bus system

\begin{tabular}{|c|c|c|c|c|c|c|}
\hline \multirow[t]{2}{*}{ Parameter } & \multirow[t]{2}{*}{ Case $1 \mathrm{~A}$} & \multirow[t]{2}{*}{ Case 1B [25] } & \multicolumn{2}{|l|}{ Case $1 \mathrm{C}$} & \multicolumn{2}{|c|}{ Case 1D } \\
\hline & & & GA & PSO & GA & PSO \\
\hline Power flow in line 1-3 (congested line) (MW) & 63.36 & 56.82 & 53.34 & 54.97 & 52.21 & 54.97 \\
\hline Real power losses (MW) & 11.834 & 14.097 & 7.8234 & 8.9453 & 5.6056 & 8.2021 \\
\hline Maximum value of voltage magnitude in p.u. & 1.0822 & 1.082 & 1.082 & 1.082 & 1.082 & 1.082 \\
\hline Minimum value of voltage magnitude in $\mathrm{p} . \mathrm{u}$. & 0.992 & 0.994 & 0.9966 & 0.9959 & 0.9956 & 0.9957 \\
\hline DG cost $(\$ / h)$ & 0 & 1800 & 1797.597 & 244.941 & 596.1 & 298.214 \\
\hline
\end{tabular}

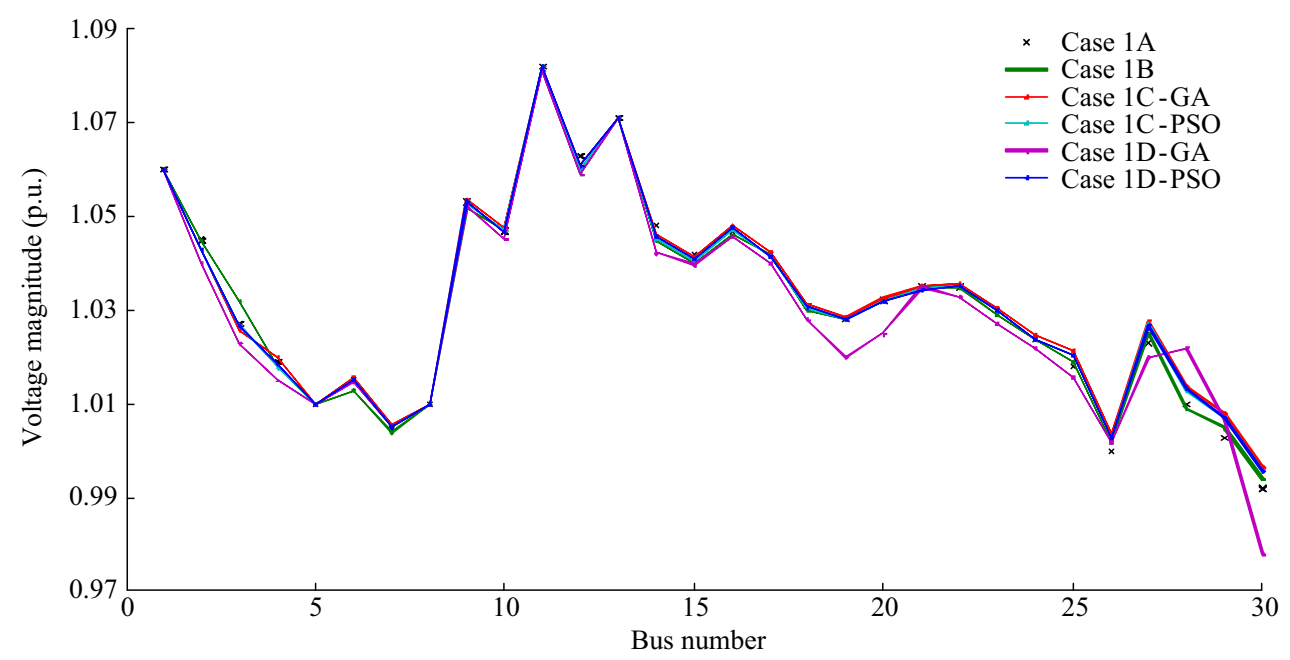

Fig. 4 Voltage profile of IEEE-30 bus system at different stages

inserting the optimum sizing of DGs according to the Cases $1 \mathrm{C}$ and $1 \mathrm{D}$.

It is also noticed that the voltage magnitudes at the load buses have been improved greatly through the multi-objective approach by PSO as compared to GA and other single objective approaches. This proves the superiority of the proposed approach as compared to GA and the approach reported in [25] for IEEE-30 bus network. Further, the proposed congestion management approach is also practiced on large test bus system to check the practical feasibility. The following sub-section discuses the various results related to the large test bus system.

\subsection{Case 2: IEEE-118 bus system}

In this sub-section, the proposed approach is applied on IEEE-118 bus system. The test system has 54 generators, 186 transmission lines and 99 loads. The total load on the system is $4242 \mathrm{MW}$. The generators cost coefficients, their maximum and minimum limits, bus and line data related to the IEEE-118 bus can be obtained from [33]. As the bus 69 is reference, generator outputs except the reference bus generator are considered as variables for the OPF run. The results of the OPF are tabulated in the Table 7 . The results of OPF obtained through proposed approach are compared with the other similar works like PSO, ALC-PSO, ICBO. The total fuel cost obtained using the proposed approach is $130062.8620(\$ / \mathrm{h})$ which is minimum as compared to GA and ICBO approaches. In Case 2A, the test system does not have DG allocation. The real power losses and power flow in the line 26-30 are 87.620 and $170.60 \mathrm{MW}$ respectively. This implies, the line 26-30 is congested when its limit is reduced from 175 to $150 \mathrm{MW}$. That means the line $26-30$ is loaded with more $13.745 \%$ of its limit. Hence, MO-PSO is implemented to alleviate the congestion in the line.

In this case $20 \%$ of total load is $848.4 \mathrm{MW}$, hence $850 \mathrm{MW}$ is considered as maximum capacity of the DG to be connected. But practically DGs are of low ratings so, in this work maximum available capacity of DG is taken as $60 \mathrm{MW}$. According to this, 14 DGs are required to be inserted in the IEEE-118 bus system to relieve congestion 
Table 7 Results of OPF according to Case 2A for IEEE-118 bus system

\begin{tabular}{|c|c|c|c|c|}
\hline Generator & $\begin{array}{l}\text { PSO } \\
(\mathrm{MW})\end{array}$ & $\begin{array}{l}\text { ALC-PSO } \\
{[33] \text { (MW) }}\end{array}$ & $\begin{array}{l}\text { ICBO } \\
{[34]} \\
(\mathrm{MW})\end{array}$ & GA (MW) \\
\hline 1 & 20.69 & 27.5202 & 370.42 & 0 \\
\hline 4 & 0 & 0 & 30.0012 & 86.897 \\
\hline 6 & 0 & 0 & 30.006 & 72.6573 \\
\hline 8 & 0 & 0 & 30.0004 & 42.2068 \\
\hline 10 & 390 & 401.6008 & 30.0017 & 14.9294 \\
\hline 12 & 86.36 & 85.5017 & 316.815 & 30.4277 \\
\hline 15 & 26.57 & 18.3415 & 68.0579 & 68.1198 \\
\hline 18 & 10.59 & 11.1001 & 30.0344 & 24.8773 \\
\hline 19 & 39.68 & 23.321 & 30.0022 & 21.392 \\
\hline 24 & 2.96 & 0 & 30.0009 & 57.654 \\
\hline 25 & 177.98 & 195.27 & 30.0031 & 100 \\
\hline 26 & 265 & 278.986 & 152.2583 & 202.6975 \\
\hline 27 & 25.13 & 15.2378 & 221.2265 & 112.208 \\
\hline 31 & 7.4 & 7.2651 & 30.0023 & 3.1887 \\
\hline 32 & 12.12 & 13.6541 & 32.1001 & 16.142 \\
\hline 34 & 17.78 & 2.4891 & 30.0468 & 55.4852 \\
\hline 36 & 27 & 8.9856 & 30.0023 & 100 \\
\hline 40 & 23 & 49.9442 & 30.0002 & 100 \\
\hline 42 & 61.23 & 42.0478 & 30.0011 & 22.7333 \\
\hline 46 & 18.35 & 19.1282 & 30.0074 & 17.4502 \\
\hline 49 & 192.53 & 193.6081 & 35.7188 & 79.6501 \\
\hline 54 & 53.61 & 50.1543 & 162.5469 & 14.6998 \\
\hline 55 & 42.97 & 31.659 & 44.4008 & 101.479 \\
\hline 56 & 26.54 & 34.7532 & 30.3515 & 100 \\
\hline 59 & 146.35 & 147.6018 & 30.0022 & 100 \\
\hline 61 & 147.23 & 149.8376 & 126.396 & 154.0434 \\
\hline 62 & 0 & 0.0022 & 123.5531 & 148.2644 \\
\hline 65 & 333.21 & 346.2842 & 30.0022 & 33.2415 \\
\hline 66 & 344.01 & 348.1853 & 290.6133 & 271.7695 \\
\hline 69 & 447.72 & 462.8761 & 290.485 & 100 \\
\hline 70 & 0 & 0 & 30.0008 & 100 \\
\hline 72 & 0 & 0 & 30.0018 & 0.0522 \\
\hline 73 & 0 & 0 & 30.0016 & 89.0752 \\
\hline 74 & 23.79 & 17.0971 & 30.0015 & 10.1664 \\
\hline 76 & 19.55 & 24.2712 & 30.0023 & 52.6154 \\
\hline 77 & 0 & 0 & 30.0046 & 16.7157 \\
\hline 80 & 424 & 416.045 & 350.109 & 528.7858 \\
\hline 85 & 0 & 0 & 30.0038 & 65.4129 \\
\hline 87 & 3.48 & 3.7122 & 31.2 & 6.4411 \\
\hline 89 & 483.78 & 505.012 & 378.9986 & 239.0351 \\
\hline 90 & 0 & 0 & 30.0003 & 116.3091 \\
\hline 91 & 0 & 0 & 30.0006 & 82.0595 \\
\hline 92 & 0 & 0 & 30.0004 & 44.4655 \\
\hline 99 & 0 & 0 & 30 & 39.6494 \\
\hline 100 & 227.52 & 232.1064 & 176.5001 & 184.1041 \\
\hline 103 & 38.56 & 39.021 & 42.0025 & 78.9053 \\
\hline
\end{tabular}

Table 7 continued

\begin{tabular}{lllll}
\hline Generator & $\begin{array}{l}\text { PSO } \\
\text { (MW) }\end{array}$ & $\begin{array}{l}\text { ALC-PSO } \\
\text { [33] (MW) }\end{array}$ & $\begin{array}{l}\text { ICBO } \\
{[34]} \\
\text { (MW) }\end{array}$ & GA (MW) \\
\hline 104 & 18.95 & 0 & 30.0007 & 40.2184 \\
105 & 12.25 & 11.5314 & 30.019 & 13.2081 \\
107 & 31.29 & 26.4823 & 30.0016 & 76.2585 \\
110 & 12.03 & 0 & 30.0008 & 18.0738 \\
111 & 34.71 & 35.9456 & 40.8017 & 0 \\
112 & 38.47 & 39.5123 & 30.0005 & 86.897 \\
113 & 6.75 & 0 & 30.0048 & 72.6573 \\
116 & 0 & 0 & 30.017 & 42.2068 \\
$\begin{array}{l}\text { Generation cost } \\
\text { (\$/h) }\end{array}$ & 130062.86 & 129546.08 & 135121.57 & 142000.00 \\
Power flow in & 170.60 & - & & 172.58 \\
line 26-30 & & & & \\
(MW) & & & & \\
\hline
\end{tabular}

Table 8 Sizing of DGs in various case studies for IEEE-118 bus system

\begin{tabular}{|c|c|c|c|c|c|}
\hline \multirow{2}{*}{$\begin{array}{l}\text { Preferable } \\
\text { locations }\end{array}$} & \multirow{2}{*}{$\begin{array}{l}\text { Case 1B } \\
(\mathrm{MW})\end{array}$} & \multicolumn{2}{|c|}{ Case 1C } & \multicolumn{2}{|c|}{ Case 1D } \\
\hline & & $\begin{array}{l}\text { GA } \\
\text { (MW) }\end{array}$ & $\begin{array}{l}\text { PSO } \\
\text { (MW) }\end{array}$ & $\begin{array}{l}\text { GA } \\
\text { (MW) }\end{array}$ & $\begin{array}{l}\text { PSO } \\
\text { (MW) }\end{array}$ \\
\hline 9 & 60 & 8.19 & 0 & 6.42 & 0.134 \\
\hline 8 & 60 & 4.26 & 0.8403 & 18.117 & 0.826 \\
\hline 10 & 60 & 5.23 & 1.9578 & 0 & 0.112 \\
\hline 30 & - & 7.61 & 1.447 & 20.166 & 0.618 \\
\hline 26 & - & 1.26 & 7.421 & 5.387 & 8.216 \\
\hline 38 & - & 3.14 & 0 & 4.322 & 0.510 \\
\hline 25 & - & 0 & 0 & 0 & 0 \\
\hline 65 & - & 0 & 0 & 7.785 & 0.620 \\
\hline 66 & - & 45.15 & 0.2234 & 12.611 & 0 \\
\hline 64 & - & 30.61 & 3.2563 & 0.8571 & 1.240 \\
\hline 61 & - & 14.22 & 0.8676 & 15.621 & 0.560 \\
\hline 67 & - & 0 & 6.7299 & 0.584 & 5.432 \\
\hline 68 & - & 30.68 & 1.3526 & 10.480 & 0.726 \\
\hline 116 & - & 2.28 & 1.3258 & 24.610 & 0.528 \\
\hline $\begin{array}{l}\text { Total DG } \\
\text { penetration }\end{array}$ & 180 & 152.63 & 25.4217 & 126.96 & 19.52 \\
\hline
\end{tabular}

in the line 26-30. The preferable locations for inserting the optimal capacity DGs with respect to the congested line 26-30 are shown in first column of Table 8.

The near optimal solutions for the TCM problem are obtained through both the GA and PSO approaches as reported. During the Case $2 \mathrm{C}$ weighting factors are set to 1 and during the Case 2D those are set to 0.25 . The results obtained in the both cases are reported in Table 8. The convergence curves for both the approaches during Cases 


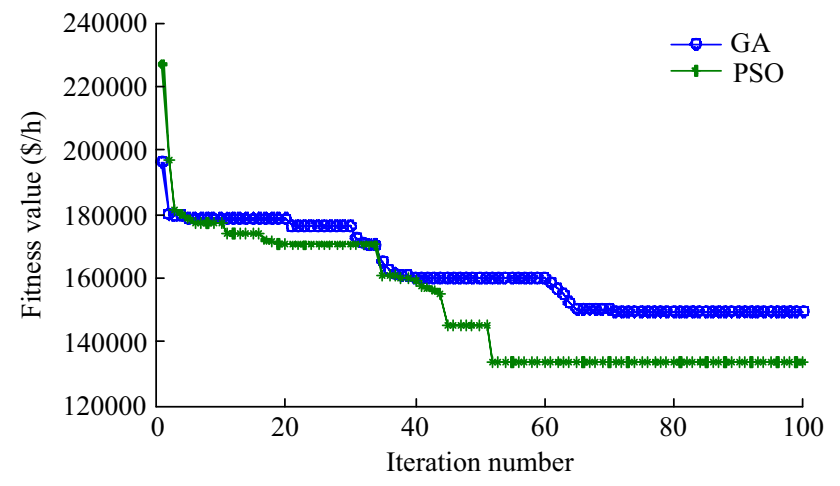

Fig. 5 Convergence curve in Case 2C for IEEE-118 bus system

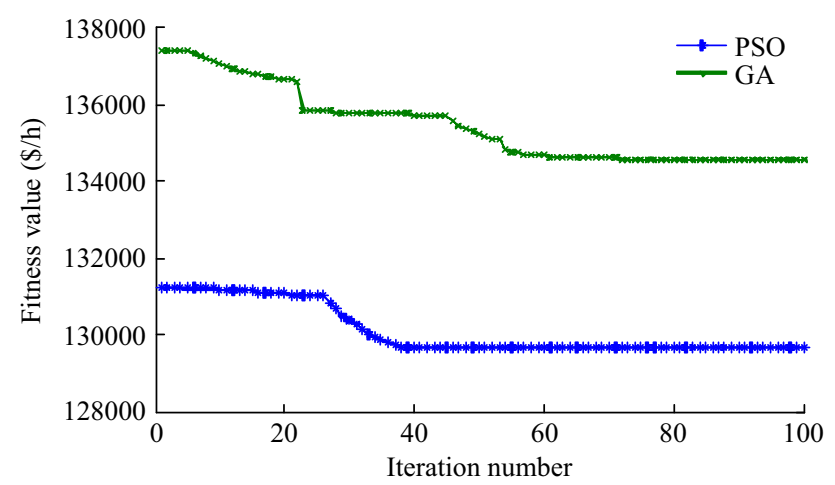

Fig. 6 Convergence curve in Case 2D for IEEE-118 bus system
2C and 2D run are shown in Figs. 5 and 6 respectively. According to Case 2B, 60 MW DGs at buses 9, 8, 10 will not alleviate the transmission line congestion. Further, the results of proposed approach are also compared with other single and multi-objective approaches using GA. This comparative analysis is shown in Table 9.

It is confirmed that, the real power losses and power flow in the congested lines have been greatly reduced with the implementation of proposed multi-objective approach using PSO. The Fig. 7 shows voltage magnitudes of each bus in p.u. before and after connecting the DGs in different cases. The figure reveals advancement in voltages after connecting the optimum sizing of DGs according to the proposed approach. It is noticed that change is not observed for the maximum voltage level, but a slight decrement is observed after connecting the optimum size of DGs according to Case 2C. The results declare the superiority of the proposed PSO approach over the GA and other single objective approaches. The results of the proposed approach also reveal that the congested lines are alleviated completely without overloading any other lines in the power system network. The entire results reveal that the power flows, real power losses and voltages have been greatly improved by inserting optimum capacity DGs by the proposed MOPSO approach.

Table 9 Comparative results of various approaches in different case studies for IEEE-118 bus system

\begin{tabular}{|c|c|c|c|c|c|c|}
\hline \multirow[t]{2}{*}{ Parameter } & \multirow[t]{2}{*}{ Case $2 \mathrm{~A}$} & \multirow[t]{2}{*}{ Case 2B [25] } & \multicolumn{2}{|c|}{ Case $2 \mathrm{C}$} & \multicolumn{2}{|c|}{ Case 2D } \\
\hline & & & GA & PSO & GA & PSO \\
\hline Power flow in line 26-30 (congested line) (MW) & 170.60 & 183.42 & 149.68 & 144.729 & 150 & 145.62 \\
\hline Real power losses (MW) & 87.620 & 102.56 & 90.12 & 87.572 & 88.16 & 84.32 \\
\hline Maximum value of voltage magnitude in p.u. & 1.0742 & 1.05 & 1.65 & 1.068 & 1.068 & 1.065 \\
\hline Minimum value of voltage magnitude in p.u. & 0.952 & 0.943 & 0.96 & 0.9519 & 0.962 & 0.974 \\
\hline DG cost $(\$ / h)$ & 0 & 5400 & 4578.9 & 762.651 & 3808.8 & 585.66 \\
\hline
\end{tabular}

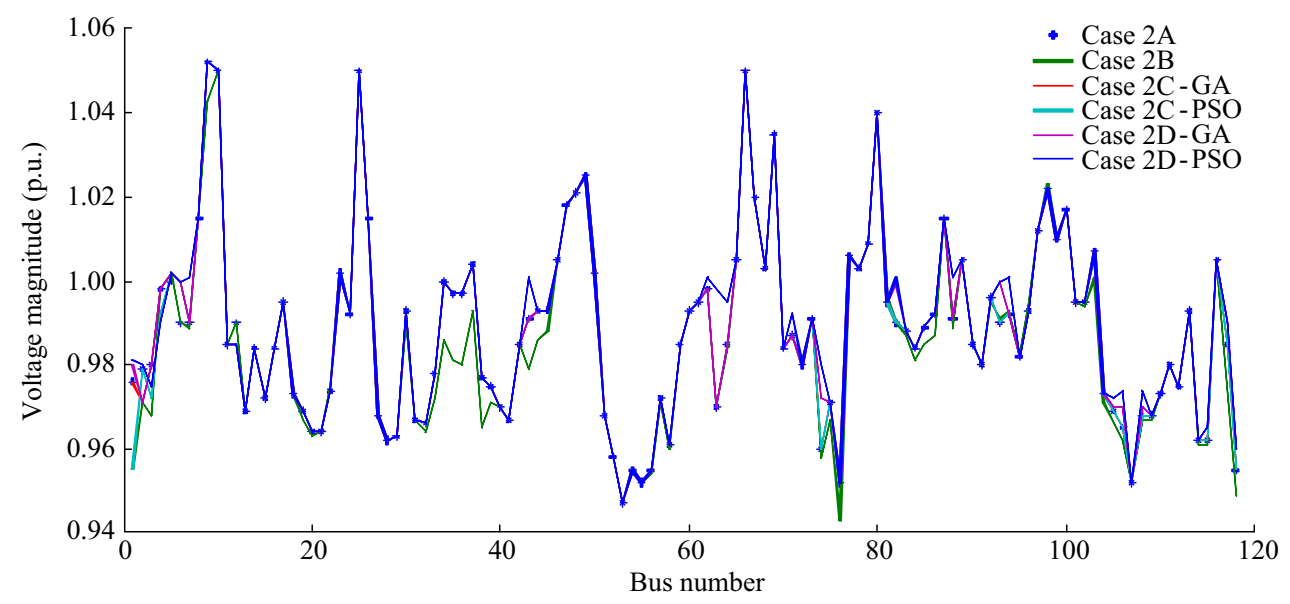

Fig. 7 Voltage profile of IEEE-118 bus system at different stages 


\section{Conclusion}

In this paper, MO-PSO based transmission congestion management problem has been proposed. Optimal sizing of renewable DGs are integrated in order to relieve the congestion in a transmission line. The multi-objective approach comprises of both the technical and economical factors like congestion, real power losses, voltage improvement and DGs investment cost respectively. The multi-objectives are made as single objective by fastening them with normalized weighting factors. Three types of renewable DGs such as solar, biomass and wind systems are integrated with the main grid at optimal locations. The feasibility of the proposed approach is checked on standard test systems like IEEE-30 bus and IEEE-118 bus test systems. It is observed that the proposed approach reduces real power losses up to 30.7 and $3.77 \%$ in the case of 30 and 118 bus test systems respectively. It was also observed that voltage profile is improved at all load buses significantly in both the test systems. The entire study depicts that the critical contingency condition has been relieved completely and a wide scope has been opened for the ISO to improve the bidding strategies in an optimal manner. Finally it can be concluded that the proposed method is efficient and feasible for solving the real time complex power system problems.

Open Access This article is distributed under the terms of the Creative Commons Attribution 4.0 International License (http:// creativecommons.org/licenses/by/4.0/), which permits unrestricted use, distribution, and reproduction in any medium, provided you give appropriate credit to the original author(s) and the source, provide a link to the Creative Commons license, and indicate if changes were made.

\section{References}

[1] Anbazhagan S, Kumarappan N (2014) Day-ahead deregulated electricity market price forecasting using neural network input featured by DCT. Energy Convers Manag 78:711-719

[2] Yadav VK, Padhy NP, Gupta HO (2011) Performance evaluation and improvement directions for an Indian electric utility. Energy Policy 39(11):7112-7120

[3] Shandilya A, Gupta H, Sharma J (1993) Method for generation rescheduling and load shedding to alleviate line overloads using local optimisation. IEE Proc C Gener Transm Distrib 140(5):337

[4] Talukdar BK, Sinha AK, Mukhopadhyay S et al (2005) A computationally simple method for cost-efficient generation rescheduling and load shedding for congestion management. Int J Electr Power Energy Syst 27(5-6):379-388

[5] Hazra J, Sinha AK (2007) Congestion management using multiobjective particle swarm optimization. IEEE Trans Power Syst 22(4):1726-1734

[6] Kumar A, Srivastava SC, Singh SN (2004) A zonal congestion management approach using real and reactive power rescheduling. IEEE Trans Power Syst 19(1):554-562
[7] Dutta S, Singh SP (2008) Optimal rescheduling of generators for congestion management based on particle swarm optimization. IEEE Trans Power Syst 23(4):1560-1569

[8] Panigrahi BK, Ravikumar Pandi V (2009) Congestion management using adaptive bacterial foraging algorithm. Energy Convers Manag 50(5):1202-1209

[9] Acharya N, Mithulananthan N (2007) Locating series FACTS devices for congestion management in deregulated electricity markets. Electr Power Syst Res 77(3-4):352-360

[10] Yorino N, El-Araby EE, Sasaki H et al (2003) A new formulation for FACTS allocation for security enhancement against voltage collapse. IEEE Trans Power Syst 18(1):3-10

[11] Huang T, Bompard E, Yan Z (2011) Congestion management impacts on bilateral electricity markets under strategic negotiation. Electr Power Syst Res 81(5):1161-1170

[12] Rodrigues AB, Da Silva MG (2003) Impact of multilateral congestion management on the reliability of power transactions. Int J Electr Power Energy Syst 25(2):113-122

[13] Esmaili M, Amjady N, Shayanfar HA (2010) Stochastic congestion management in power markets using efficient scenario approaches. Energy Convers Manag 51(11):2285-2293

[14] Xu D, Girgis AA (2001) Optimal load shedding strategy in power systems with distributed generation. In: 2001 IEEE power engineering society winter meeting. Conference proceedings (Cat. No.01CH37194). IEEE, pp 788-793

[15] Esmaili M, Chaktan E, Ali H (2014) Optimal placement of distributed generations considering voltage stability and power losses with observing voltage-related constraints. Appl Energy 113:1252-1260

[16] Gampa SR, Das D (2015) Optimum placement and sizing of DGs considering average hourly variations of load. Int J Electr Power Energy Syst 66:25-40

[17] Murty VVSN, Kumar A (2014) Electrical power and energy systems mesh distribution system analysis in presence of distributed generation with time varying load model. Int J Electr Power Energy Syst 62:836-854

[18] Aman MM, Jasmon GB, Bakar AHA et al (2014) A new approach for optimum simultaneous multi-DG distributed generation units placement and sizing based on maximization of system loadability using HPSO (hybrid particle swarm optimization) algorithm. Energy 66:202-215

[19] Kayal P, Chanda CK (2015) Optimal mix of solar and wind distributed generations considering performance improvement of electrical distribution network. Renew Energy 75:173-186

[20] Mohamed Imran A, Kowsalya M, Kothari DP (2014) A novel integration technique for optimal network reconfiguration and distributed generation placement in power distribution networks. Int J Electr Power Energy Syst 63:461-472

[21] Mohamed IA, Kowsalya M (2014) Optimal size and siting of multiple distributed generators in distribution system using bacterial foraging optimization. Swarm Evolut Comput 15: $58-65$

[22] Gil Mena AJ, Martín García JA (2015) An efficient approach for the siting and sizing problem of distributed generation. Int $\mathrm{J}$ Electr Power Energy Syst 69:167-172

[23] Rosseti GJS, De Oliveira EJ, De Oliveira LW et al (2013) Optimal allocation of distributed generation with reconfiguration in electric distribution systems. Electr Power Syst Res 103: $178-183$

[24] Kanwar N, Gupta N, Niazi KR et al (2015) Optimal distributed generation allocation in radial distribution systems considering customer-wise dedicated feeders and load patterns. J Mod Power Syst Clean Energy 3(4):475-484. doi:10.1007/s40565-0150169-0

[25] Singh K, Yadav VK, Padhy NP et al (2014) Congestion management considering optimal placement of distributed generator 
in deregulated power system networks. Electr Power Compon Syst 42(1):13-22

[26] Singh AK, Parida SK (2013) Congestion management with distributed generation and its impact on electricity market. Int $\mathrm{J}$ Electr Power Energy Syst 48(1):39-47

[27] Yesuratnam G, Thukaram D (2007) Congestion management in open access based on relative electrical distances using voltage stability criteria. Electr Power Syst Res 77(12):1608-1618

[28] Afkousi-Paqaleh M, Abbaspour-Tehrani Fard A, Rashidinejad M (2010) Distributed generation placement for congestion management considering economic and financial issues. Electr Eng 92(6):193-201

[29] Gaing Z-L (2004) A particle swarm optimization approach for optimum design of PID controller in AVR system. IEEE Trans Energy Convers 19(2):384-391

[30] Cao J, Yan Z, Xu X et al (2016) Optimal power flow calculation in AC/DC hybrid power system based on adaptive simplified human learning optimization algorithm. J Mod Power Syst Clean Energy 92(4):193-201. doi:10.1007/s40565-016-0227-2

[31] Borges CLT, Falcão DM (2006) Optimal distributed generation allocation for reliability, losses, and voltage improvement. Int $\mathbf{J}$ Electr Power Energy Syst 28(6):413-420

[32] Kennedy J, Eberhart R (1995) Particle swarm optimization. In: Proceedings of ICNN'95-international conference on neural networks. Perth, WA, Australia, 27 November-1 December 1995, IEEE, pp 1942-1948
[33] Pratap R, Mukherjee V, Ghoshal SP (2016) Particle swarm optimization with an aging leader and challengers algorithm for the solution of optimal power flow problem. Appl Soft Comput J 40:161-177

[34] Bouchekara HREH, Chaib AE, Abido MA et al (2016) Optimal power flow using an Improved Colliding Bodies Optimization algorithm. Appl Soft Comput J 42:119-131

Rajagopal PEESAPATI received his M.Tech degree from National Institute of Technology Jamshedpur, India. Presently he is pursuing Ph.D. degree from the same institute. His research interests include power system planning and restructuring.

Vinod Kumar YADAV received his M.Tech degree from National Institute of Technology Jamshedpur and Ph.D. degree from Indian Institute of Technology Roorkee. His research interests include power system restructuring and operation.

Niranjan KUMAR received his M.Tech degree from National Institute of Technology Jamshedpur and Ph.D. degree from Indian Institute of Technology Roorkee. His research interests include power system restructuring and AC/DC power flow analysis. 